\title{
Paired box gene 2 is associated with estrogen receptor $\alpha$ in ovarian serous tumors: Potential theory basis for targeted therapy
}

\author{
MIN WANG and HAIFEN MA \\ Department of Pathology, Beilun People's Hospital, Ningbo, Zhejiang 315800, P.R. China
}

Received November 4, 2015; Accepted April 21, 2016

DOI: $10.3892 / \mathrm{mco} .2016 .935$

\begin{abstract}
It has been suggested that Paired box gene $(\mathrm{PAX}) 2$ is activated by estradiol via estrogen receptor (ER) $\alpha$ in breast and endometrial cancer. The expression of PAX2 was restricted to ovarian serous tumors and only one case was positive in borderline mucinous tumor in our previous study. In the present study, immunohistochemistry was performed to assess the expression of ER $\alpha$ in 58 cases of ovarian serous tumors, including 30 serous cystadenomas, 16 borderline serous cystadenomas, 12 serous carcinomas and 67 cases of ovarian mucinous tumors, including 29 mucinous cystadenoma, 23 borderline mucinous cystadenoma and 15 mucinous carcinoma, which were the same specimens with detection of PAX 2 expression. The results demonstrated that ER $\alpha$ was expressed in $10 \%(3 / 30)$ of serous cystadenomas, $62.5 \%(10 / 16)$ borderline serous cystadenomas and $66.7 \%(8 / 12)$ serous carcinomas. The expression of ER $\alpha$ in borderline serous cystadenomas and serous carcinomas were significantly higher compared with that in serous cystadenomas $(\mathrm{P}<0.01)$. ER $\alpha$ was detected in $3.4 \%(1 / 29)$ mucinous cystadenoma, $26.1 \%(6 / 23)$ borderline mucinous cystadenoma and only $6.7 \%$ (1/15) mucinous carcinoma. Furthermore, a scatter plot of the expression of PAX 2 and ER $\alpha$ revealed a linear correlation between them in ovarian serous tumors $(\mathrm{P}<0.0001)$. With few positive results, no correlation was determined in ovarian mucinous tumors. It was demonstrated that PAX2 is associated with ER $\alpha$ in ovarian serous tumors, and this may become a potential theory basis for targeted therapy for ovarian serous tumors. Further research is required to determine how PAX2 and ER $\alpha$ work together, and the role of targeted therapy in ovarian serous tumors.
\end{abstract}

Correspondence to: Dr Haifen Ma, Department of Pathology, Beilun People's Hospital, 1288 Lushan East Road, Ningbo, Zhejiang 315800, P.R. China

E-mail: mhfen@163.com

Key words: ovarian serous tumors, immunohistochemistry, paired box gene 2 , estrogen receptor $\alpha$, correlation

\section{Introduction}

Ovarian tumor is the most common female tumor type. Epidemiological data indicates a steady rise of the incidence rate in Europe and Asia. In the USA, the mortality of ovarian cancer is $\sim 67.7 \%$ annually (1). The histological type of most ovarian tumors is serous ovarian tumor. Ovarian serous carcinomas account for $70 \%$ of all ovarian carcinomas. Therefore, further research on ovarian tumors is required so that treatment can be successful. Paired box gene (PAX)2 belongs to the PAX family, consisting of nine members, PAX1-9. The genes of the PAX family have a conserved DNA sequence motif, which comprises a 128 amino acid domain in the amino-terminal portion of the protein (2). The role of PAX2 is crucial to embryogenic development, morphogenesis and organogenesis (3). PAX2 overexpression is associated with tumor types, including renal cell carcinoma, breast cancer and ovarian cancer (4-6). Estrogens influence numerous physiological processes in mammals, which mediates its effects through the estrogen receptor (ER). The ER exists in two predominant forms, $E R \alpha$ and $E R \beta$. ER $\alpha$ is used as a therapeutic target and the presence of ER $\alpha$ may be effective for endocrine therapy of tumors. It has been previously reported that PAX2 is activated by estradiol via ER $\alpha$ in breast cancer (7). It was also reported that PAX2 was a common target of estrogen- and tamoxifen (ER agonist)-bound ER $\alpha$ and can promote the growth of endometrial cancer cells (8). Therefore, a correlation may exist between PAX2 and ER $\alpha$ in ovarian tumors, which little attention has been paid to. In the present study, the expression of $\mathrm{ER} \alpha$ was detected in ovarian tumor types and the correlation between PAX2 and ER $\alpha$ was assessed.

\section{Materials and methods}

Patients and tissue samples. A total of 58 patients with ovarian serous tumor types, including serous cystadenomas $(n=30)$, borderline serous cystadenomas $(n=16)$, serous carcinomas $(\mathrm{n}=12)$ and patients of ovarian mucinous tumors $(n=67)$, including mucinous cystadenoma $(n=29)$, borderline mucinous cystadenoma $(n=23)$, mucinous carcinoma $(n=15)$, were from the Beilun People's Hospital of Ningbo (Zhejiang, China). Patients who received no pre-operative chemoradiation treatment and had post-operative diagnosis as ovarian tumors were randomly selected for the present study. All specimens were the same as used in our previous study (9). It 
A
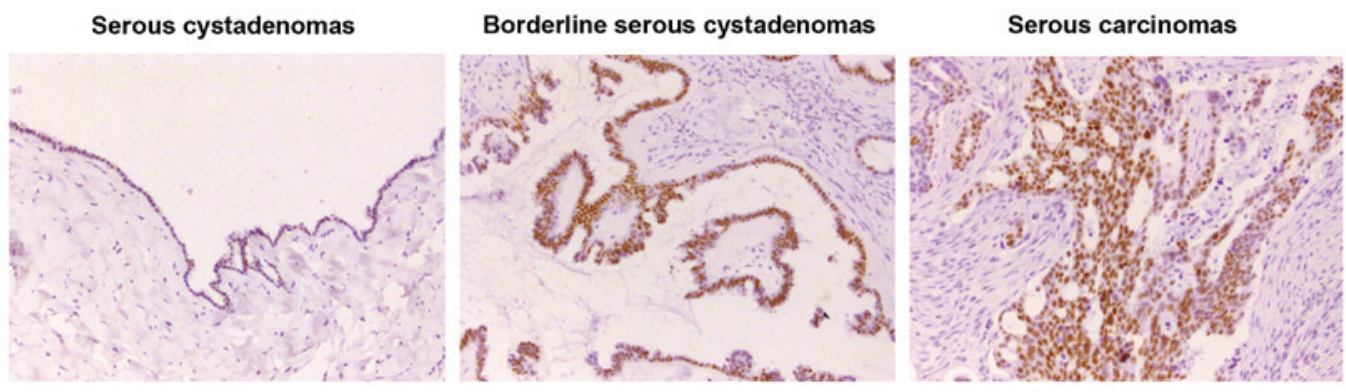

B

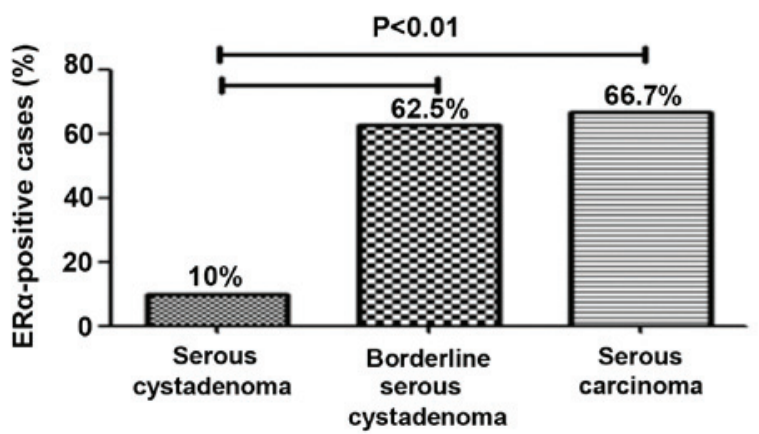

Figure 1. ER $\alpha$ expression in ovarian serous tumors. (A) The expression patterns of ER $\alpha$ in serous cystadenoma, borderline serous cystadenoma and serous carcinoma were assessed by immunohistochemistry (magnification, x200). (B) A comparison of the expression of ER $\alpha$ was performed between the ovarian serous tumors. The percentage of positive ER $\alpha$ cells in serous cystadenoma were significantly lower compared with borderline serous cystadenoma and serous carcinoma. The data are presented as the positive cases and rates $(\mathrm{P}<0.01)$. ER, estrogen receptor.

should be noted that one of ovarian mucinous tumors in our previous study was not included in the present study due to a lack of tissue. The samples were collected and $10 \%$ neutral formalin-fixed following surgery and paraffin-embedded following dehydration. Hematoxylin and eosin staining and immunohistochemical analysis was used on the basis of the availability of archived paraffin-embedded tissue blocks. Ethical approval was obtained from the hospital and informed consent was obtained from all patients prior to the study. Tumor histological type was based on currently used histopathologic criteria and the histological characteristics were reviewed by two pathologists in a blinded manner.

Immunohistochemistry. The ER $\alpha$ levels were measured by immunohistochemical analysis. Briefly, the specimens were sectioned ( $4 \mu \mathrm{m}$ thick) using a paraffin slicing machine, mounted onto poly-L-lysine-coated glass slides and allowed to dry at $65^{\circ} \mathrm{C}$ for $30-60 \mathrm{~min}$. The slides were subsequently deparaffinized in xylene and transferred through three changes of $95 \%$ ethanol. The samples were then transferred into water. For antigen retrieval, the slides were boiled in a pressure cooker at maximum heat for $3 \mathrm{~min}$ containing $0.01 \mathrm{~mol} / \mathrm{l}$ sodium citrate $(\mathrm{pH} 6.0)$ and cooling for $30 \mathrm{~min}$ at room temperature. Endogenous peroxidase activity was inhibited in $0.3 \% \mathrm{H}_{2} \mathrm{O}_{2}$ for $8 \mathrm{~min}$ at room temperature. Following incubation, the slides were washed three times in phosphate-buffered saline (PBS) for $2 \mathrm{~min}$. The slides were incubated primary antibodies against rabbit anti-ER $\alpha$ (cat. no. ab37438; Abcam, Cambridge, UK) at 1:100 dilution for $1 \mathrm{~h}$ at $37^{\circ} \mathrm{C}$. After washing three times in PBS for 2 min each, the bound primary antibody was detected using a ready-to-use secondary antibody kit (cat. no. K5007; Dako, Carpinteria, CA, USA) for $30 \mathrm{~min}$ at room temperature and the chromogenic substrate 3,3-diaminobenzidine. The slides were washed in distilled water, counterstained with hematoxylin, dehydrated and mounted with permanent media.

The appropriate positive and negative controls were included in the sections. Expression levels of ER $\alpha$ were evaluated by counting at least 500 tumor cells in representative high-power fields. Only tumor cells with nuclear staining were considered positive for ER $\alpha$. Tumor cell percentage was scored as follows: $0=<1 \%$ positive; $1=\geq 1$ and $<10 \%$ positive; $2=10-75 \%$ positive; $3=>75 \%$ positive. Scoring criteria for staining intensity was as follows: $0=$ no stainin $\mathrm{g} ; 1$ =weak; 2 = moderate; $3=$ strong staining. The staining index was evaluated as the product of the percentage of positive tumor cells and staining intensity score. Using this method of estimation, the expression of ER $\alpha$ in the tumors was evaluated by determining the staining index with scores of $0,1,2,3,4,6$ or 9 , and '-' for 0 or 1 , '+' for 2 , ' ++ ' for 3 or 4 , ' +++ ' for 6 or 9. In statistical analysis, ER $\alpha$ was considered positive with a value of a $>2$.

Statistical analysis. Statistical analyses were performed using SPSS 19.0 software (IBM SPSS, Chicago, IL, USA). The $\chi^{2}$-test was used to compare the expression of ER $\alpha$ with various histological types. Linear regression was used to analyze the association between PAX2 and ER $\alpha$ in ovarian serous tumor types. $\mathrm{P}<0.05$ was considered to indicate a statistically significant difference.

\section{Results}

Expression of ERa in ovarian serous tumor types. Immunohistochemical analysis revealed the expression of $E R \alpha$ in ovarian serous tumors. It was shown in Fig. 1A 
Table I. Expression levels of PAX2 and ER $\alpha$ in ovarian serous tumors.

\begin{tabular}{|c|c|c|c|c|c|c|c|c|c|c|}
\hline \multirow[b]{2}{*}{ Tumor type } & \multicolumn{5}{|c|}{ PAX2 expression } & \multicolumn{5}{|c|}{ ER $\alpha$ expression } \\
\hline & - & + & ++ & +++ & $\mathrm{n}(\%)$ & - & + & ++ & +++ & $\mathrm{n}(\%)$ \\
\hline Serous cystadenoma & 0 & 10 & 10 & 10 & $30 / 30(100)$ & 27 & 2 & 1 & 0 & $3 / 30(10)$ \\
\hline Borderline serous cystadenoma & 0 & 0 & 5 & 11 & $16 / 16(100)$ & 6 & 1 & 9 & 0 & $10 / 16(62.5)$ \\
\hline Serous carcinoma & 0 & 1 & 5 & 6 & $12 / 12(100)$ & 4 & 2 & 5 & 1 & $8 / 12(66.7)$ \\
\hline
\end{tabular}

PAX, Paired box gene 2; ER, estrogen receptor.

Table II. Expression levels of PAX2 and ER $\alpha$ in ovarian mucinous tumors.

\begin{tabular}{|c|c|c|c|c|c|c|c|c|c|c|}
\hline \multirow[b]{2}{*}{ Tumor type } & \multicolumn{5}{|c|}{ PAX2 expression } & \multicolumn{5}{|c|}{ ER $\alpha$ expression } \\
\hline & - & + & ++ & +++ & $\mathrm{n}(\%)$ & - & + & ++ & +++ & $\mathrm{n}(\%)$ \\
\hline Mucinous cystadenoma & 29 & 0 & 0 & 0 & $0 / 29(0)$ & 28 & 1 & 0 & 0 & $1 / 29(3.4)$ \\
\hline Borderline mucinous cystadenoma & 22 & 0 & 1 & 0 & $1 / 23(4)$ & 17 & 2 & 2 & 2 & $6 / 23(26.1)$ \\
\hline Mucinous carcinoma & 15 & 0 & 0 & 0 & $0 / 15(0)$ & 14 & 0 & 1 & 0 & $1 / 15(6.7)$ \\
\hline
\end{tabular}

PAX, Paired box gene 2; ER, estrogen receptor.

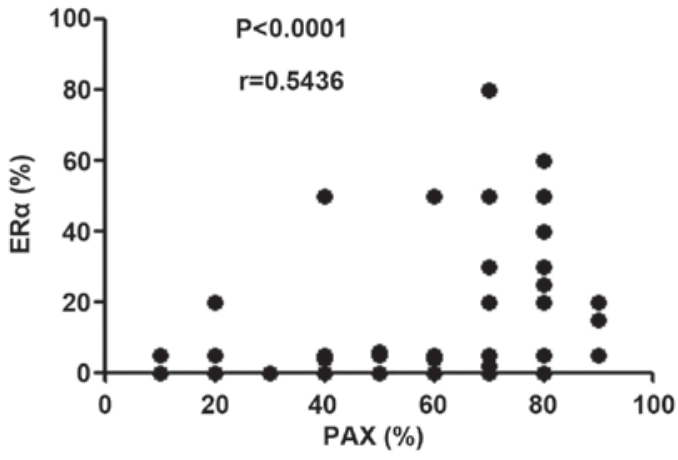

Figure 2. Correlation plot of the expression levels of PAX2 and ER $\alpha$ in 58 cases of ovarian serous tumors $(\mathrm{r}=0.5436 ; \mathrm{P}<0.0001)$. PAX, Paired box gene 2; ER, estrogen receptor.

that $10 \%(3 / 30)$ in serous cystadenomas, $62.5 \%(10 / 16)$ in borderline serous cystadenomas and $66.7 \%(8 / 12)$ in serous carcinomas. The expression of ER $\alpha$ in borderline serous cystadenomas and serous carcinomas were significantly higher compared with in serous cystadenomas $(\mathrm{P}<0.01)$. However, no difference between borderline serous cystadenomas and serous carcinomas was observed ( $\mathrm{P}>0.05$; Fig. 1B; Table I).

Expression of ER in ovarian mucinous tumor types. The present study additionally assessed the expression levels of ER $\alpha$ in 67 cases of ovarian mucinous tumors by immunohistochemistry. Expression of ER $\alpha$ was detected in 3.4\% (1/29) mucinous cystadenoma, 26.1\% (6/23) borderline mucinous cystadenoma and only $6.7 \%$ (1/15) mucinous carcinoma (Table II).

Expression of PAX2 in ovarian tumor types. In our previous study, it was demonstrated that the expression of PAX2 was restricted to all 58 ovarian serous tumor types (Table I). By contrast, only one sample was positive in 68 mucinous tumors from the same patients (Table II) (9).

Correlation between the expression levels of PAX2 and ER $\alpha$. Furthermore, a correlation plot of the expression levels of $\mathrm{PAX} 2$ and $\mathrm{ER} \alpha$ in all 58 ovarian serous tumor samples revealed that there is a linear correlation between them $(r=0.5436$; $\mathrm{P}<0.0001$; Fig. 2). This confirmed that the expression of PAX2 is proportional to the expression of $\mathrm{ER} \alpha$ in ovarian serous tumors. By contrast, with few positive results, no correlation was determined in ovarian mucinous tumor samples.

\section{Discussion}

Ovarian cancer is one of the most fatal cancer types in females, the most common histological type being serous carcinoma. The pathogenesis of serous carcinoma remains to be completely understood and an effective treatment strategy is required. In the present case series, both 58 cases of ovarian serous tumors and 67 cases of mucinous tumors consisting of three groups, including cystadenomas and borderline cystadenomas and carcinomas. Immunohistochemistry was performed to assess the expression of PAX2 and ER $\alpha$ in ovarian serous tumors and mucinous tumors. Our previous results revealed that PAX2 was expressed in $100 \%$ ovarian serous tumors (9). Other researchers revealed PAX2 expression in $\geq 60 \%$ serous carcinomas $(10,11)$. The difference between the results may be associated with the number of samples assessed. The present data indicated that PAX2 may involved in the occurrence of ovarian serous tumors.

No doubt estrogens are significant in the development, growth, invasion and metastasis of breast, ovarian and 
endometrial cancer. O'Donnell et al (12) identified that estrogen-driven growth of epithelial ovarian carcinoma is mediated by the activation of ER $\alpha$-, but not ER $\beta$-mediated transcription. Hu et al (13) found that the mRNA and protein expression levels of ER $\alpha$ increased in ovarian carcinoma compared with normal ovarian tissues. Estrogen stimulates the growth of ovarian tumor cell lines by ER $\alpha$ (14). In the present study, the expression of ER $\alpha$ observed in borderline cystadenomas and carcinomas were significantly higher compared with cystadenomas in ovarian serous tumors, which indirectly confirmed the results of previous studies (12-14).

Previously a few results indicated that PAX2 was activated by estradiol via ER $\alpha$ in breast and endometrial cancer. Beauchemin et al (7) concluded from their study that PAX2 may serve a novel role in the maintenance of a low invasive behavior in luminal breast cancer cells upon exposure to estradiol, and the overexpression and activation of PAX2 in these cells was sufficient to reduce their invasive ability (7). Hurtado et al (15) suggested that endocrine resistance in breast cancer may results due to PAX2 being a crucial mediator of ER repression of Avian erythroblastic leukemia viral oncogene homolog (ERBB)2 by the anti-cancer drug tamoxifen. PAX2 silencing was able to abrogate the inhibition of ERBB2 transcription and increases ERBB2-dependent cell proliferation (15). Additionally, a previous study determined that PAX2 was a downstream target of ER $\alpha$ in endometrial cells, and PAX2 was a common target of estrogen- and tamoxifen (ER agonist)-bound ER $\alpha$ and may promote the growth of endometrial cancer cells (8).

In addition, ER $\alpha$ is used as a therapeutic target for target organs of endocrine hormone. Tamoxifen is a selective estrogen receptor modulator with anti-estrogenic activity in the breast. As adjuvant hormone therapy, it has a clear beneficial effect in patients with breast cancer. However, tamoxifen shows partial estrogenic activity in the uterus and its use has been associated with an increased incidence of endometrial cancer (16).

However, little attention has been given to ovarian tumor types regarding correlation between PAX2 and ER $\alpha$. In the present study of 58 ovarian serous tumor samples, a linear correlation was observed between the expression levels of PAX2 and ER $\alpha$. It may become a potential theory basis for targeted therapy for ovarian serous tumor types. Further research is required to determine how PAX2 and ER $\alpha$ work together, and the role of targeted therapy with tamoxifen in ovarian serous tumors.

\section{Acknowledgements}

The present study was supported by the Clinical Research Fund Project of Zhejiang Province (no. 2013CYC-A77)

\section{References}

1. Jemal A, Siegel R, Ward E, Hao Y, Xu J and Thun MJ: Cancer statistics, 2009. CA Cancer J Clin 59: 225-249, 2009.

2. Eccles MR, He S, Legge M, Kumar R, Fox J, Zhou C, French M and Tsai RW: PAX genes in development and disease: The role of PAX2 in urogenital tract development. Int J Dev Biol 46: 535-544, 2002.

3. Lang D, Powell SK, Plummer RS, Young KP and Ruggeri BA: PAX genes: Roles in development, pathophysiology, and cancer. Biochem Pharmacol 73: 1-14, 2007.

4. Gnarra JR and Dressler GR: Expression of Pax-2 in human renal cell carcinoma and growth nhibition by antisense oligonucleotides. Cancer Res 55: 4092-4098, 1995.

5. Silberstein GB, Dressler GR and Van Horn K: Expression of the PAX2 oncogene in human breast cancer and its role in progesterone-dependent mammary growth. Oncogene 21: 1009-1016, 2002.

6. Tung CS, Mok SC, Tsang YT, Zu Z, Song H, Liu J, Deavers MT, Malpica A, Wolf JK, Lu KH, et al: PAX2 expression in low malignant potential ovarian tumors and low-grade ovarian serous carcinomas. Mod Pathol 22: 1243-1250, 2009.

7. Beauchemin D, Lacombe C and Van Themsche C: PAX2 is activated by estradiol in breast cancer cells of the luminal subgroup selectively, to confer a low invasive phenotype. Mol Cancer 10: 148, 2011.

8. Wu H, Chen Y, Liang J, Shi B, Wu G, Zhang Y, Wang D, Li R, Yi X, Zhang $\mathrm{H}$, et al: Hypomethylation-linked activation of PAX2 mediates tamoxifen-stimulated endometrial carcinogenesis. Nature 438: 981-987, 2005.

9. Wang M, Ma H, Pan Y, Xiao W, Li J, Yu J and He J: PAX2 and PAX8 reliably distinguishes ovarian serous tumors from mucinous tumors. Appl Immunohistochem Mol Morphol 23: 280-287, 2015.

10. Gao FF, Krasinskas AM and Chivukula M: Is PAX2 a reliable marker in differentiating diffuse malignant mesotheliomas of peritoneum from serous carcinomas of müllerian origin? Appl Immunohistochem Mol Morphol 20: 272-276, 2012.

11. Tong GX, Chiriboga L, Hamele-Bena D and Borczuk AC: Expression of PAX2 in papillary serous carcinoma of the ovary: Immunohistochemical evidence of fallopian tube or secondary Müllerian system origin? Mod Pathol 20: 856-863, 2007.

12. O'Donnell AJ, Macleod KG, Burns DJ, Smyth JF and Langdon SP: Estrogen receptor-alpha mediates gene expression changes and growth response in ovarian cancer cells exposed to estrogen. Endocr Relat Cancer 12: 851-866, 2005.

13. Hu Z, Zhu L, Tan M, Cai M, Deng L, Yu G, Liu D, Liu J and Lin B: The expression and correlation between the transcription factor FOXP1 and estrogen receptors in epithelial ovarian cancer. Biochimie 109: 42-48, 2015.

14. Cunat S, Hoffmann P and Pujol P: Estrogens and epithelial ovarian cancer. Gynecol Oncol 94: 25-32, 2004.

15. Hurtado A, Holmes KA, Geistlinger TR, Hutcheson IR, Nicholson RI, Brown M, Jiang J, Howat WJ, Ali S and Carroll JS: Regulation of ERBB2 by oestrogen receptor-PAX2 determines response to tamoxifen. Nature 456: 663-666, 2008.

16. Machado F, Rodríguez JR, León JP, Rodríguez JR, Parrilla JJ and Abad L: Tamoxifen and endometrial cancer. Is screening necessary? A review of the literature. Eur J Gynaecol Oncol 26: $257-265,2005$ 